\title{
Information aus der Fachkommission für Dopingbekämpfung (FDB) von Swiss Olympic Association
}

\author{
M. Kamber, M. Strupler für die Fachkommission für Dopingbekämpfung
}

\section{Neue Dopingliste ab 1. Januar 2004}

Am 3. März 2003 wurde in Kopenhagen das AntiDopingprogramm der Welt-Anti-Doping-Agentur (WADA) von allen Delegierten der Sportverbände und Regierungen angenommen. Das Internationale Olympische Komitee (IOK) wie auch Swiss Olympic haben dem Programm zugestimmt. Das Programm umfasst den Code, vier Standards und Beispiele bester Praxis. Der Code und die vier Standards müssen zwingend angewendet werden. Einer der Standards ist die Liste der Dopingmittel und -methoden. Sie wurde erstmals von der entsprechenden Kommission der WADA erarbeitet und trat auf den 1. Januar 2004 in Kraft. Der Hauptzweck der Liste ist die Festlegung der verbotenen Substanzen und Methoden im Sport im Rahmen der Regeln des Codes.

\section{Neue Einteilung}

Die Liste wurde mit drei neuen Substanzklassen erweitert. Es betrifft dies die Cannabinoide, Beta2-Agonisten und Glukokortikoide. Die Diuretika sind neu unter der Klasse der maskierenden Substanzen aufgeführt. Cannabinoide waren bisher nur in bestimmten Sportarten verboten, sie sind ab 2004 in allen Sportarten im Wettkampf verboten. Beta-2-Agonisten wurden früher unter Stimulantien und Anabolika geführt, sie bilden ab 2004 eine eigenständige Klasse. Für die Glukokortikoide gilt wie bisher die Regelung, dass jede orale, rektale, intravenöse und intramuskuläre Anwendung im Wettkampf verboten ist. Neu müssen für alle Sportarten alle anderen diesbezüglich erlaubten Anwendungen vor dem Wettkampf gemeldet werden.

Neu ist auch, dass die bisherige Klausel «und verwandte Substanzen» wegfällt. Sie wurde abgelöst durch spezifischere Ausdrücke bei den einzelnen Substanzklassen.

Die bisher unter den in bestimmten Sportarten verbotenen Substanzklassen aufgeführten Lokalanästhetika sind vollständig von der Liste gestrichen und unterliegen somit keinen Einschränkungen mehr.
Ebenfalls neu ist, dass nur noch gewisse Grenzwerte (z.B. für Ephedrin, Cathin, Salbuta$\mathrm{mol}$ ) in der Liste genannt werden. Andere Grenzwerte (wie z.B. für Cannabinoide, Morphin, Nandrolon) finden sich hingegen nur noch im internationalen Standard für die Labors.

\section{Änderungen bei den verbotenen Substanzen}

\section{Stimulanzien}

Bei den Stimulanzien ist die wichtigste Änderung, dass schwache Stimulanzien wie Koffein, Phenylpropanolamin und Pseudoephedrin von der Liste gestrichen wurden. Diese Substanzen werden aber zwecks Überwachung weiterhin von den Labors analysiert. Neu auf der Liste werden namentlich folgende Substanzen genannt: Adrafinil, Amfetaminil, Benzfentamin, Dimethylamfetamin, Furfenorex, Methylamfetamin und Modafinil.

\section{Narkotika}

Die folgenden Narkotika sind neu namentlich auf der Liste: Hydrocodon, Oxycodon und Oxymorphon. Zudem wird kein Hinweis auf verwandte Substanzen mehr gemacht, so dass die Aufzählung in dieser Substanzklasse abschliessend ist.

\section{Anabolika}

Neu wird die Substanzklasse in «anabole, androgene Steroide» (AAS) und «andere anabol wirkende Substanzen» aufgeteilt. Bei den AAS werden neu namentlich genannt: Boldion, Delta1-Androsten-3,17-dion, Drostandiol, 4-Hydroxytestosteron, 4-Hydroxy-19-nortestosteron, Mestenolon, Oxandrolon, Quinbolon, Stenbolon und 1-Testosteron (delta1-dihydro-testosteron). Neu ist auch die Aufteilung der AAS in exogene und endogene AAS samt Umschreibung der Massnahmen bei einem abweichenden Laborbefund.

Unter «andere anabol wirkende Substanzen» werden Clenbuterol und neu Zeranol verboten. Die bisher unter dieser Klasse aufgeführten anderen Beta-2-Agonisten sind in einer eigenen Substanzklasse zusammengefasst. 


\section{Änderung beim Meldewesen}

Wie oben erwähnt, sind neben dem AntiDoping-Code der WADA auch vier technische Standards in Kraft. Neben der Dopingliste ist vor allem der internationale Standard für Ausnahmebewilligungen zu therapeutischen Zwecken (ATZ) (International Standard for Therapeutic Use Exemption, TUE) bei der Betreuung von Sportlerinnen und Sportlern wichtig. Dieses Reglement sieht vor, den Prozess zur Gewährung einer Ausnahmebewilligung für die Verwendung von verbotenen Substanzen zu therapeutischen Zwecken zu harmonisieren. In Ausnahmefällen, bei denen keine anderen alternativen Therapien möglich sind, kann ein Antrag für die Verwendung verbotener Substanzen oder Methoden gestellt werden. Der Antrag hat in der Regel mindestens 21 Tage vor der geplanten Therapie zu erfolgen.

Es gibt zwei Möglichkeiten, eine Ausnahmebewilligung zu erhalten:

- einfaches Verfahren für Beta-2-Agonisten und lokal oder topisch applizierte Glukokortikoide;

- reguläres Verfahren für alle anderen verbotenen Substanzen.

Beim Einfachen Verfahren kann der Athlet ein Arztzeugnis auf dem offiziellen (seit 1. Januar 2004 neuen) Formular von Swiss Olympic an die Geschäftsstelle der Fachkommission einsenden. Das Zeugnis hat sofort Gültigkeit. Die Sportlerin oder der Sportler erhält keine Bestätigung, sollte aber von der behandelnden Fachperson eine Kopie des Antrages erhalten. Das Zeugnis für die Beta-2-Agonisten ist während eines Jahres gültig, wobei der ausstellende Arzt die Diagnose mit den gängigen pneumologisch-spirometrischen Untersuchungen absichern muss. Diese Untersuchungsergebnisse müssen jedoch nicht mehr eingereicht, können aber zur Überprüfung der Bewilligung verlangt werden.

Nach dem gleichen Verfahren können auch lokal, topisch oder intraartikulär applizierte Glukokortikoide gemeldet werden. Die systemische Verwendung dieser Substanzen ist im Wettkampf weiterhin verboten.

Das Reguläre Verfahren gilt für alle anderen Anträge zur Verwendung von verbotenen Substanzen zu therapeutischen Zwecken. Das ausführliche Formular kann auf der Geschäftsstelle verlangt oder von den unten aufgeführten Websites heruntergeladen werden. Das ausgefüllte Formular muss mit allen nötigen Unterlagen an die Geschäftsstelle eingesandt werden. Die Fachkommission hat eine ärztliche Kommission eingesetzt, welche über diese Anträge entscheidet. Der Athlet erhält innert drei Wochen eine schriftliche Bewilligung oder einen negativen Entscheid.

Für beide Verfahren gilt, dass die Bestätigungen respektive die Bewilligungen an die entsprechenden internationalen Verbände und an die WADA weitergeleitet werden müssen. Die offiziellen Formulare zum Einreichen der Anträge sind von der Welt-Anti-Doping-Agentur vorgegeben. Die Fachkommission hat lediglich die Ergänzungen in deutscher Sprache vorgenommen.

Zur Erinnerung: die neusten Informationen zum Thema Doping sind auf www.dopinginfo. ch zu finden! 